\title{
Microscopy and molecular biology for the diagnosis and evaluation of malaria in a hospital in a rural area of Ethiopia
}

Maria A Santana-Morales ${ }^{1 *}$, Raquel N Afonso-Lehmann ${ }^{1}$, Maria A Quispe ${ }^{1}$, Francisco Reyes ${ }^{3}$, Pedro Berzosa $^{2}$, Agustin Benito $^{2}$, Basilio Valladares ${ }^{1}$ and Enrique Martinez-Carretero ${ }^{1}$

\begin{abstract}
Background: Malaria is a leading public health problem in Ethiopia. Accurate diagnosis of Plasmodium infections is crucial for the reduction of malaria in tropical areas and for epidemiological studies. The role of light microscopy (LM) as gold standard has been questioned and, therefore, new molecular methods have been developed for the detection of Plasmodium species. The aim of the present work was to compare different malaria diagnostic methods in order to detect the most common species of Plasmodium and to broaden the knowledge of malaria prevalence in a hospital in a rural area in Ethiopia.
\end{abstract}

Methods: A cross-sectional survey of 471 individuals was carried out in a hospital in the rural area of Gambo (Ethiopia). Blood samples were prepared for microscopic observation and collected in filter paper for SeminestedMultiplex PCR (SnM-PCR) and real time PCR (qPCR) testing. The SnM-PCR was considered as the gold standard technique and compared with the rest. Thus, agreement between SnM-PCR and LM was determined by calculating Kappa Statistics and correlation between LM and qPCR quantification was calculated by pair-wise correlation co-efficient.

Results: Samples analysed by LM and SnM-PCR were positive for Plasmodium sp. 5.5\% and 10.5\%, respectively. Sensitivity was $52.2 \%$ by LM and $70 \%$ by qPCR. Correlation co-efficient between microscopy counts and qPCR densities for Plasmodium vivax was $\mathrm{R}^{2}=0.586$. Prevalence was estimated at $7 \%$ ( $95 \% \mathrm{Cl}$ : 4.7-9.3). Plasmodium vivax was the dominant species detected and the difference was statistically significant $\left(x^{2}=5.121 p<0.05\right)$. The highest prevalence of the parasite (10.9\%) was observed in age groups under 15 years old.

Conclusion: Accurate malaria diagnostic methods have a great effect in the reduction of the number of malaria-infected individuals. SnM-PCR detection of malaria parasites may be a very useful complement to microscopic examination in order to obtain the real prevalence of each Plasmodium species. Although SnM-PCR shows that it is a good tool for the determination of Plasmodium species, today light microscopy remains the only viabletool for malaria diagnosis in developing countries. Therefore, re-inforcement in the training of microscopists is essential for making the correct diagnosis of malaria. Plasmodium vivax was the predominant species in Gambo, a meso-endemic area for this species.

Keywords: Diagnosis, Prevalence, Malaria, Ethiopia

\footnotetext{
*Correspondence: msanmor@ull.es

${ }^{1}$ University Institute of Tropical Diseases and Public Health of the Canary Islands University of La Laguna, Tenerife, Spain

Full list of author information is available at the end of the article
} 


\section{Background}

Malaria is the most prevalent tropical infectious disease in the world. An estimated 300-500 million people are annually infected with malaria, resulting in 1.5-3 million deaths [1]. Malaria mostly affects children in highly endemic areas with stable transmission. In areas with low or moderate endemicity, all age groups are affected and such areas are at a special risk of severe epidemics $[2,3]$.

In Ethiopia, malaria is a leading public health problem where an estimated $68 \%$ of the population lives in malarious areas and three-quarters of the total land mass is regarded as malarious [4]. Plasmodium falciparum and Plasmodium vivax are the two predominant malaria species, accounting for $60 \%$ and $40 \%$ of malaria cases, respectively [5]. Malaria transmission follows a seasonal pattern (September-November), depending on the altitude and rainy season. Moreover, epidemic malaria is common, particularly in the highlands $(1,000-2,000 \mathrm{~m}$ above sea level). However, many areas in the south and west of the country have a rainfall season beginning earlier in March and May or have no clearly defined rainfall season [5].

Therefore, accurate diagnosis of Plasmodium infections is crucial to reduce morbidity and mortality in tropical areas [6]. Studies of epidemiology and immunity depend on accurate detection, diagnosis, and density estimation.

Traditionally, light microscopy (LM) examination of blood smears has been considered the gold standard for the diagnosis of malaria [7]. LM has clear advantages since it incurs low costs, allows species identification and quantification and neither complex sample preparation nor advanced technology is required [8]. However, the role of LM as gold standard has been questioned due to false negative results at low levels of parasitaemia, with a predicted limit of detection of five to 20 parasites per microlitre of blood and frequent errors in species identification in mixed infections $[9,10]$. In this sense, microscopy seems to be an imperfect reference standard. Therefore, it is difficult to estimate its true sensitivity and specificity or to evaluate new diagnostic methods [11]. Several molecular methods based on the amplification of DNA have been developed for the detection of malarial infections in humans [12-16]. The semi-nested multiplex malaria PCR (SnM-PCR) is a widely used method and it is considered a molecular gold standard due to its good performance in the detection of mixed species infection and the ability to differentiate the four species of Plasmodium $[15,16]$. In recent years, new molecular methods have been developed for the detection of Plasmodium species, mostly based on real time quantitative PCRs (qPCR) [17,18]. These new molecular methods have been promoted as an automated, quantitative, and closed system that reduces the risk of cross- contamination inherent in conventional PCR [19]. Several real-time PCR methods for malaria have been described and validated within a research setting with high sensitivity and specificity values [20]. However, some advantages should be considered such as their ability to detect mixed Plasmodium infections but also some limitations, such as their application in rural areas without adequate laboratory conditions [19].

Alternative methods sensitive enough to detect low levels of parasitaemia in asymptomatic infections are required to complement or replace parasitological examination with light microscopy [21]. This would allow minimization of errors in diagnosis (false positives, false negatives and species misidentification) that may lead to biased estimates of protective efficacy against the parasite. The incorporation of molecular tools for the characterization of parasite infections has allowed an increase sensitivity in the detection of human malarial parasites in blood [22].

The aim of the present study was to compare different malaria diagnostic methods and to broaden the knowledge of malaria prevalence in a hospital in Gambo, Ethiopia.

\section{Methods}

\section{Study site and cross-sectional survey}

The study took place at Gambo General Rural Hospital. The hospital is located in the province of West Arsi, Ethiopia, which lies at $7^{\circ} 18^{\prime} 20.82^{\prime \prime}$ north latitude and $38^{\circ}$ $48^{\prime} 55.37^{\prime \prime}$ east longitude. The study was carried out from January to May 2010 and was approved by the Ethical Committee of Gambo General Rural Hospital.

A sample of 306 adults aged $\geq 16$ years and 165 children aged $\leq 15$ years was randomly selected from Gambo General Rural Hospital patients. The samples were divided in 192 women whose mean age was 32 years (range 16 to 89 years), 114 men, whose mean age was 40 years (range 16 to 98 years) and 165 children, whose mean age was five years (range one to 15 years). Pregnant women were excluded due to hospital policy. Microscopy was performed at the laboratory of the above-mentioned hospital and PCRs were performed at the University Institute of Tropical Diseases and Public Health of the Canary Islands.

\section{Laboratory methods}

Blood sample collection and light microscopy observation

Finger-prick blood samples were collected and both thick and thin blood smears were prepared for microscopic observation. During the study period temperature values were around $25^{\circ} \mathrm{C}$; samples were dried at room temperature before storage and were shopped at $4^{\circ} \mathrm{C}$ for further analysis. Thin and thick films were stained with a $10 \%$ Giemsa solution. A minimum of 200 microscopic 
fields were examined at a magnification of 1,000X using oil immersion optics before a slide was declared negative for malaria parasites by LM. Parasitaemia per microlitre of blood was estimated from the thick films as follows: the number of parasites per 200 white blood cells was multiplied by 8,000 (an average white blood cell count per microlitre) and then divided by 200 . Routinely slides were read twice. Discordant results were evaluated by a third slide reading. Final species diagnosis was based on the majority agreement between experienced microscopists at Gambo General Rural Hospital.

Finger-prick samples on 3MM filter paper were obtained for the PCR assays. Each filter paper specimen was stored in a plastic bag at room temperature and shipped to the Canary Islands.

\section{DNA template extraction and amplification}

DNA extraction was performed using commercial kits (Speedtools tissue DNA Extraction Kit, Biotools,Madrid, Spain). The seminested PCR was considered as the molecular gold standard. Therefore, suspected Plasmodium species by LM were confirmed by semi-nested-multiplex PCR [22] and by qPCR using primers and probes developed by commercial kits (PrimerDesign ${ }^{\mathrm{TM}}$ Ltd, Alicante, Spain), which amplify the plasmepsin 4 gene in P. falciparum and the aspartic protease PM4 gene in P.vivax. Each reaction contained $5 \mu \mathrm{L}$ DNA, 10 $\mu \mathrm{L} 2 x$ Precision ${ }^{\mathrm{TM}}$ MasterMix (Primerdesign), $1 \mu \mathrm{L}$ Pathogen Primer/ Probe mix, $1 \mu \mathrm{L}$ Internal extraction control primer/probe mix and $3 \mu \mathrm{L}$ RNAse/DNAse free water. The thermal profile used was 15 minutes at $37^{\circ} \mathrm{C}$, followed by 10 minutes at $95^{\circ} \mathrm{C}$ and 50 cycles of 10 seconds at $95^{\circ} \mathrm{C}$ and 1 minute at $60^{\circ} \mathrm{C}$. A second qPCR was performed as previously described by Rosanas-Urgell et al. [6]. All PCRs were performed two times for each sample.

\section{qPCR validation}

Evaluation of qPCR efficiency and reproducibility was performed on standard curves using two positive control plasmids with the respective inserted amplicons available with the commercial kit. Geometric mean and standard deviation were calculated from triplicates in three independent assays. The standard curve for each Plasmodium species was made from a 10 -fold serial dilution of the control plasmids ranging from $20^{5}$ copies $/ \mu \mathrm{L}$ to 2 copies $/ \mu \mathrm{L}$. Amplification efficiencies for the different primer pairs and probes were calculated with the formula: Efficiency $=10(-1 /$ Slope $)-1$.

The amount of target in an unknown sample was quantified by converting the threshold cycle $(\mathrm{Ct})$ into template copy number by using the two standard curves. Samples yielding Ct values equal or higher than 40, were considered negative for Plasmodium species [23]. All protocols were performed following manufacturers instructions two times.

\section{Statistical analysis}

Results from SnM-PCR were compared with those from LM and qPCR. In this study, SnM-PCR was used as a gold standard. Agreement between SnM-PCR and LM was determined by calculating Kappa Statistics with 95\% confidence intervals. Values were interpreted with the Landis and Koch classification [24] as follows: $k=0.41-$ 0.60 , moderate agreement, $\mathrm{k}=0.61-0.80$, good agreement; $\mathrm{k}=0.81-1.00$, almost perfect agreement beyond chance. Correlation between LM and qPCR quantification was calculated by pair-wise correlation co-efficient.

Prevalence was calculated by dividing the number of positive samples by the total number of tested samples. The age trends of the different categorical variables were assessed using the Chi-square test for linear trend.

All statistical calculations were performed with SpSS19 statistical software and $P$ values $<0.05$ were considered statistically significant.

\section{Results}

The study included 417 samples from Gambo General Rural Hospital. Samples analysed by LM and SnM-PCR were positive for Plasmodium sp. $5.5 \%$ and $10.5 \%$, respectively. None of the parasite positive samples by microscopy was negative by SnM-PCR while $5 \%$ of the negative samples by LM were positive by SnM-PCR. Overall LM sensitivity was only $52.2 \%$, being $58.8 \%$ for $P$. vivax and $33.3 \%$ for P. falciparum (Table 1). In children, the sensitivity was $66.6 \%$ for $P$. vivax and $20 \%$ for $P$. falciparum. The concordance between both microscopy and SnM-PCR by the Kappa co-efficient showed a good agreement $(k=0.62)$.

All SnM-PCR-positive samples were analysed by qPCR. Overall, sensitivity was $70 \%$, being $82.8 \%$ for $P$. vivaxand $33.3 \%$ for $P$. falciparum (Table 1 ).

The geometric mean parasite density was 2,274 parasites $/ \mu \mathrm{L}$ (range 680 to 8,000 parasites $/ \mu \mathrm{L}$ ) by microscopy. By qPCR (PrimerDesign ${ }^{\mathrm{TM}} \mathrm{Ltd}$ ) the geometric mean parasite density was 5.6 copies/ $\mu$ l of blood (ranging from 0.78 to 279.23 copies/ $\mu \mathrm{l}$ of blood). Correlation co-efficient between microscopy counts and qPCR densities for $P$. vivax was $\mathrm{R}^{2}=0.586$ (Figure 1). Positive samples were analysed by the GPCR designed by

Table 1 Comparative study of the sensitivity of the LM and qPCR using SnM-PCR as gold standard

\begin{tabular}{llll}
\hline & Sensitivity & & \\
\cline { 2 - 4 } & Total & P. vivax & P.falciparum \\
\hline LM & $52.2 \%$ & $58.8 \%$ & $33.3 \%$ \\
\hline qPCR & $70 \%$ & $82.3 \%$ & $33.3 \%$ \\
\hline
\end{tabular}




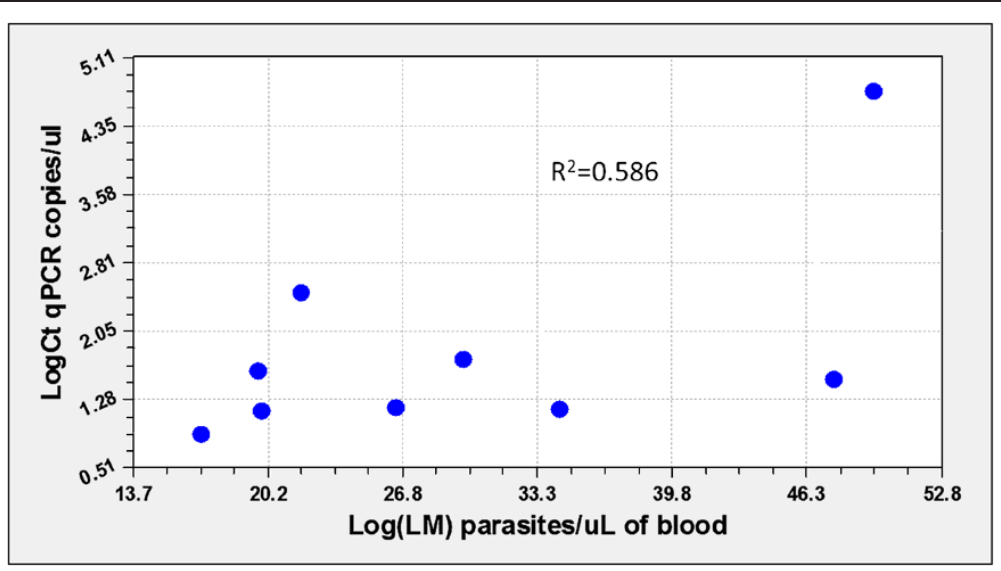

Figure 1 Comparison of quantification assessed by $L M$ versus $\mathrm{qPCR}\left(\right.$ PrimerDesign $\left.^{\mathrm{TM}} \mathrm{Ltd}\right)$.

Rosanas-Urgell et al. [6]. The correlation co-efficient between both qPCRs in $P$. vivax was $\mathrm{R}^{2}=0,980$. These correlation co-efficients could not be estimated for $P$. falciparum since only ten samples were positive by light microscopy.

A total of 471 samples, selected as defined above, were included in the cross-sectional malaria study. Based on PCR results, weighted parasite prevalence was estimated at 7\% (95\% CI: 4.7-9.3). The prevalence of $P$. falciparum was $2.1 \%$ (95\% CI: $0.81-3.4$ ) while that of $P$. vivax was 5\% (95\% CI: 2.9-6.7). Plasmodium vivax was the dominant species detected and the difference was statistically significant $\left(\chi^{2}=5.121 p<0.05\right)$. Malaria prevalence in children was lower than in adults and the difference was statistically significant $\left(\chi^{2}=5.93 p<0.05\right)$ (Table 2 ).

Among tested adult individuals, infection frequently occurred before 30 years of age and only in three adults were parasitized at an age over 30 years old. The highest prevalence of the parasite $(10.9 \%)$ was observed in age groups under 15 years old with a mean age of five years (Figure 2).

\section{Discussion}

Ethiopia includes regions of differing malaria endemicity and transmission. The peak of malaria incidence follows the main rainfall season (July-September) each year. However, many areas in the south and west of the country have a rainfall season beginning earlier in March and May or have no clearly defined rainfall season [25].

Table 2 Malaria prevalence by sex and age

\begin{tabular}{lccccc}
\hline Plasmodium species identified Children & Female & Male & $\mathbf{X}^{2}$ & \multicolumn{1}{l}{$\boldsymbol{P}$-value } \\
\hline P. falciparumN(\%) & $6(3.6)$ & $4(2.1)$ & 0 & - & - \\
\hline P. vivaxN(\%) & $12(7.3)$ & $8(4.2)$ & $3(2.6)$ & - & - \\
\hline Total $\mathrm{N}(\%)$ & $18(10.9)$ & $12(6.25)$ & $3(2.6)$ & 5.93 & 0.01 \\
\hline
\end{tabular}

Futhermore, the sample collection was carried out during the dry season in this area.

A SnM-PCR was considered and used in this study as a molecular gold standard. The prevalence obtained by SnM-PCR was 7\%, higher than the estimated by LM method, which demonstrates an increased sensitivity with respect to the latter. In this regard, it is noteworthy that all microscopy-positive samples were also found to be positive by SnM-PCR. However, the SnM-PCR test was able to detect positive samples that were missed by microscopy.

In this study a high number of children (80\%), who initially had been negative for LM method, were later found to be infected with $P$. falciparum by SnM-PCR. These undetected sub-microscopic infections do not develop the disease in the patients but they may have an enormous impact on malaria transmission in endemic areas. Infected individuals may play the role of parasite reservoir since their blood is able to infect mosquito vectors and they may reintroduce malaria into certain regions [26,27]. Molecular methods have potential use to detect malaria parasites in asymptomatic infections,

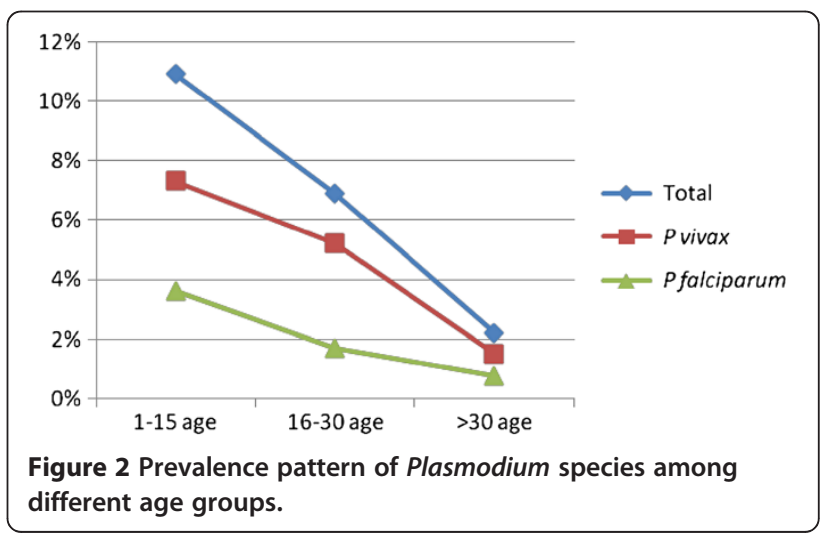


mainly in children infected with $P$. falciparum, which causes the most severe malaria infection.

The qPCR sensitivity was $70 \%$ when compared to SnM-PCR. Despite measurements taken over 45 cycles in $\mathrm{qPCR}$, previous studies and the manufacturers consensus rule was considered to decide whether a sample was positive or not (Ct value $<40$ [23]).

The qPCR is the most analytically sensitive method (sensitivity 70\%), followed by microscopy (sensitivity $52.5 \%$ ), when compared to SnM-PCR. Overall, qPCR shows substantial agreement with other molecular techniques for the detection of Plasmodium prevalence. The SnM-PCR has been reported for the diagnosis of lowlevel parasitaemia, and real-time PCR for the diagnosis of high-level parasitaemia. In countries where malaria is not endemic and where it has recently re-emerged, there is lack of physicians and microscopy-skilled laboratory staff specializing in malaria, which makes the diagnosis of malaria difficult. Therefore, SnM-PCR is a good alternative since it does not require special training for interpretation of the results, the opposite to microscopy, in which specific training is needed for species differentiation. In addition, SnM-PCR is not altered by the subjectivity of the observer [22].

The advantage of qPCR to other molecular techniques is the quantification of parasite densities. The correlation between quantifications by both qPCR showed a high correlation $\left(R^{2}=0.980\right)$ confirming that $\mathrm{qPCR}$ using commercial kits (PrimerDesign ${ }^{\mathrm{TM}}$ Ltd) is an accurate method to quantify parasite densities. When correlating quantification by qPCR with LM counts in samples where both techniques showed positive results, a low correlation for $P$. vivax $\left(\mathrm{R}^{2}=0.586\right)$ was found. This low value might be due to overall lower densities, possibly around the detection limit in $P$. vivax infections, because the scarcity of the template in case of a very low parasite density is expected to lead to imperfect detection [6]. Another possible explanation would be the presence of schizonts in the blood, because clinical samples with similar levels of parasitaemia by LM but with different proportions of schizonts (14-21 genomes) will vary in copy number and consequently in real-time PCR parasite quantification. In this sense, the comparison of parasitaemia determined by LM with that assessed by genomic standard DNA quantification curve showed significantly divergent results among malaria samples [28].

Microscopic diagnosis is considered to be the reference standard for determining the protective efficacy of prophylactic drugs or vaccines. However, microscopy is an imperfect reference standard with many inherent limitations [29]. The difficulties in correctly identifying lowdensity infection may contribute to the discrepancy in microscopy results. Furthermore, these discrepancies indicate a need to implement a rigorous quality assurance system within the routine laboratory diagnostic techniques for malaria in Ethiopia. Molecular techniques, such as PCR, have a lower detection threshold for Plasmodium than microscopy [30,31], and it may be a more sensitive diagnostic tool in a population where low-density infections are expected. For this reason, the study addressing malaria situation in Gambo was carried out using SnM-PCR.

The prevalence observed was $7 \%$, which is higher than the prevalence observed in previous surveys performed in Amhara (4.1\%), Jimma (5.2\%) and SNNP Regional States (5.4\%) [32-34]. This is due to the fact that the prevalence value was obtained with SnM-PCR, which is a more sensitive technique than microscopy. The prevalence of Plasmodium species was $69.7 \%$ and $30.3 \%$ for $P$. vivax and $P$. falciparum, respectively, unlike the previous paradigm of Plasmodium species composition in Ethiopia (P. falciparum $60 \%$ and P. vivax $40 \%$ of total malaria cases) [5]. These differences seem to indicate that the proportion of both species is changing. A study, carried out from 2007 to May 2009. It is also important to highlight the fact that this study was carried out with patients admitted to a hospital in a rural area and thus this was a biased study. In the area of study a decrease in cases of $P$. falciparum mono-infection and an increase of $P$. vivax mono-infection have been previously reported [35] and another study, conducted in areas of Oromia in December 2009, found a greater proportion of Plasmodium infection due to P. vivax [36]. The result of the present study, carried out between January and May 2010, may be explained due to the tendency of $P$. vivax to cause long-term chronic infection [37] and to dominate during low transmission periods and dry season (March-June) [38].

In the present study, a significant association between children patients and number of positive blood smears $\left(\chi^{2}=5.93 p<0.05\right)$ was found. Where transmission is relatively stable and intense, the mean and median age of patients diagnosed with malaria at clinics is generally under five years old [39]. For this reason, due to such age-dependency of the infection, children acquire certain malaria immunity [40]. Studies reported that individuals living in areas of unstable and low intensity malaria transmission do not acquire significant immunity to the disease, and hence malaria infections can be observed in all age groups [41,42]. In the present study, the least affected age group was the group over 30 years old (9.1\%). The epidemiological condition prevailing in Gambo from a prospective parasitological survey point of view suggests that the area is characteristic of a stable, moderate level of malaria endemicity being considered a meso-endemic area, based on the classification established by WHO [43]. 


\section{Conclusion}

As might be expected, in the present study, both PCR detection protocols were more efficient methods than microscopic examination. SnM-PCR methodology seems to be the most sensitive method to detect $P$. falciparum and $P$. vivax from clinical blood spots dried on filter paper, avoiding losing $80 \%$ of infection by $P$. falciparum. This large difference $(80 \%$ of positive individuals who were negative by light microscopy) suggests that microscopists are not always appropriately trained in the diagnosis of malaria. Therefore it is necessary to implement quality systems in laboratories to avoid inter-laboratory variability.

These results suggest that, in malaria endemic areas where transmission of both $P$. falciparum and $P$. vivax occurs, SnM-PCR detection of malaria parasites may be a very useful complement to microscopic examination in order to obtain the real prevalence of each species and accurate epidemiological data. The correct diagnosis of malaria parasite species can reduce the number of malaria-infected individuals who carry the parasites among populations.

The results of the present study also contribute to evaluate and broaden the knowledge of malaria in Gambo. Results of the cross-sectional study show 7\% prevalence and a higher prevalence of $P$. vivax versus $P$. falciparum. In addition, this study shows a greater number of infected children compared to adults, suggesting that Gambo is a meso-endemic area.

\section{Competing interests}

The authors declare that they have no competing interests.

\section{Authors' contributions}

MASM carried out the sample collection in Gambo (Ethiopia), the microscopy and molecular study, the analysis and interpretation of data and prepared the manuscript. RNAL and MAQ helped with the statistical analysis and interpretation. PB and $A B$ helped with the performance of molecular studies. FR helped with the collection of samples. BV helped to draft the manuscript and EMC coordinated and funded the study and drafted the manuscript. All authors read and approved the final manuscript.

\section{Acknowledgements}

The authors would like to acknowledge the Spanish Society of Tropical Medicine and International Health (SEMTSI) for supporting an International SEMTSI 2009 fellowship and the laboratory staff of the Gambo General Rural Hospital for their support in collecting the data. This survey was funded by the Tropical Disease Cooperative Research Network (RICET) and the University Institute of Tropical Diseases and Public Health of the Canary Islands.

\section{Author details}

${ }^{1}$ University Institute of Tropical Diseases and Public Health of the Canary Islands University of La Laguna, Tenerife, Spain. ${ }^{2}$ National Centre of Tropical Medicine, Institute of Health Carlos III, Madrid, Spain. ${ }^{3}$ Gambo General Rural Hospital, Shashemane, Ethiopia.

Received: 11 April 2012 Accepted: 30 May 2012

Published: 13 June 2012

\section{References}

1. WHO: World Health Organization Expert Committee on Malaria. WHO Technical report series N 892. Geneva, Switzerland: Geneva, Switzerland; 2000.
2. Molineaux L: The epidemiology of human malaria as an explanation of its distribution, including some implications for its control. In Malaria: Principles and Practice of Malariology. Volume 2. Edited by Wernsdorfer WH, McGregor I. London: Churchill Livingston; 1988:969-970.

3. MacDonald G: The Epidemiology and Control of Malaria. London, New York, and Toronto: Oxford University Press; 1957.

4. Cambell CC: Challenges facing malaria therapy in Africa. J Infect Dis 1991, 163:1207-1211.

5. Ministry of Health: Malaria and other vector borne diseases prevention and control team. National Five Years Strategic Plan for Malaria Control in Ethiopia. Addis Ababa: $\mathrm{MOH} ; 2001$

6. Rosanas-Urgell A, Mueller D, Betuela I, Barnadas C, Iga J, Zimmerman PA, del Portillo HA, Silba P, Mueller I, Felger I: Comparison of diagnostic methods for the detection and quantification of the four sympatric Plasmodium species in field samples from Papua New Guinea. Malar J 2010, 9:361.

7. WHO: World malaria report 2008. Geneva, Switzerland: World Health OrganizationPress; 2008.

8. Moody AH, Chiodini PL: Non-microscopic method for malaria diagnosis using OptiMAL IT, a second-generation dipstick for malaria pLDH antigen detection. Br J Biomed Sci 2002, 59:228-231.

9. Coleman RE, Sattabongkot J, Promstaporm S, Maneechai N, Tippayachai B, Kengluecha A, Rachapaew N, Zollner G, Miller RS, Vaughan JA, Thimasarn K, Khuntirat B: Comparison of PCR and microscopy for the detection of asymptomatic malaria in a Plasmodium falciparum/vivax endemic area in Thailand. Malar J 2006, 5:121.

10. Ochola LB, Vounatsou P, Smith T, Mabaso ML, Newton CR: The reliability of diagnostic techniques in the diagnosis and management of malaria in the absence of a gold standard. Lancet Infect Dis 2006, 6:582-588.

11. Valenstein PN: Evaluating diagnostic tests with imperfect standards. Am J Clin Path 1990, 93:252-258.

12. Kain KC, Lanar DE: Determination of genetic variation within Plasmodium falciparum by using enzymatically amplified DNA from filter paper disks impregnated with whole blood. J ClinMicrobiol 1991, 29:1171-1174

13. Lal AA, Changkasiri S, Hollingdale MR, McCutchan TF: Ribosomal RNAbased diagnosis of Plasmodium falciparum malaria. Mol Biochem Parasitol 1989, 36:67-72.

14. Singh B, Cox-Singh J, Miller AO, Abdullah MS, Snounou G, Rahman HA: Detection of malaria in Malaysia by nested polymerase chain reaction amplification of dried blood spots on filter papers. Trans $R$ Soc Trop Med Hyg 1996, 90:519-521.

15. Snounou G, Pinheiro L, Gonçalves A, Fonseca L, Dias F, Brown KN, Do Rosario $V$ : The importance of sensitive detection of malaria parasites in the human and insect hosts in epidemiological studies, as shown by the analysis of field samples from Guinea Bissau. Trans $R$ Soc Trop Med Hyg 1993, 87:649-653.

16. Snounou G, Viriyakosol S, Zhu XP, Jarra W, Thaithong S, Brown KN: Identification of the four human malaria parasite species in field samples by the polymerase chain reaction and detection of high prevalence of mixed infections. Mol Biochem Parasitol 1993, 58:283-292.

17. Lee MA, Tan CH, Aw LT, Tang CS, Singh M, Lee SH, Chia HP, Yap EP: Rea time fluorescence-based PCR for detection of malaria parasites. J Clin Microbiol 2002, 40:4343-4345.

18. Farcas GA, Zhong KJ, Mazzulli T, Kain KC: Evaluation of the RealArt Malaria LC real-time PCR assay for malaria diagnosis. J Clin Microbiol 2004, 42:636-638.

19. Shokoples SE, Ndao M, Kowalewska-Grochowska K, Yanow SK: Multiplex real-time PCR assay for discrimination of Plasmodium species with improved sensitivity for mixed infections. J Clin Microbiol 2009, 47(Suppl 4):975-980

20. Erdman LK, Kain KC: Molecular diagnostic and surveillance tools for global malaria control. Travel Med Infect Dis 2008, 6:82-99.

21. Luz K, Vasquez D, Zalis MG, Daniel-Ribeiro CT, Alecrim W, Ferreira-daCruz MF: Standardization of a very specific and sensitive single PCR for detection of Plasmodium vivax in low parasitized individuals and its usefulness for screening blood donors. Parasitol Res 2006, 98:519-524

22. Rubio JM, Benito A, Roche J, Berzosa PJ, Garcia ML, Mico M, Edu M, Alvar J: Semi-nested, multiplex polymerase chain reaction for detection of 
human malaria parasites and evidence of Plasmodium vivax infection in Equatorial Guinea. Am J Trop Med Hyg 1999, 60:183-187.

23. Bustin SA: Why the need for qPCR publication guidelines?-The case for MIQE. Methods 2010, 50:217-226.

24. Landis JR, Koch GG: An application of hierarchical kappa-type statistics in the assessment of majority agreement among multiple observers. Biometrics 1977, 33:363-374.

25. Graves PM, Richards FO, Ngondi J, Emerson PM, Shargie EB, Endeshaw T, Ceccato $P$, Ejigsemahu $Y$, Mosher AW, Hailemariam A, Zerihun M, Teferi T, Ayele B, Mesele A, Yohannes G, Tilahun A, Gebre T: Individual, household, and environmental risk factors for malaria infection in Amhara, Oromia and SNNP regions of Ethiopia. Trans R Soc Trop Med Hyg 2009, 103:1211-1220.

26. Vinetz JM, Gilman RH: Asymptomatic Plasmodium parasitemia and the ecology of malaria transmission. Am J Trop Med Hyg 2002, 66:639-640

27. Alves FP, Gil LHS, Marrelli MT, Ribolla PEM, Camargo EP, Silva LHP: Asymptomatic carriers of Plasmodium spp. as infection source for malaria vector mosquitoes in the Brazilian amazon. J Med Entomol 2005, 42:777-779.

28. Gama BE, Silva-Pires F, Lopes M, Cardoso MA, Britto C, Torres KL, Lima LM, Souza JM, Daniel-Ribeiro CT, Ferreira-da-Cruz MF: Real-time PCR versus conventional PCR for malaria parasite detection in low-grade parasitemia. ExpParasitol 2007, 116:427-432

29. Payne D: Use and limitations of light microscopy for diagnosing malaria at the primary health care level. Bull World Health Organ 1988, 66:621-626.

30. Harris I, Sharrock WW, Bain LM, Gray KA, Bobogare A, Boaz L, Lilley K, Krause D, Vallely A, Johnson ML, Gatton ML, Shanks GD, Cheng Q: A large proportion of asymptomatic Plasmodium infections with low and submicroscopic parasite densities in the low transmission setting of Temotu Province, Solomon Islands: challenges for malaria diagnostics in an elimination setting. Malar J 2010, 9:254

31. Okell LC, Ghani AC, Lyons E, Drakeley CJ: Submicroscopic infection in Plasmodium falciparum-endemic populations: A systematic review and meta-analysis. J Infect Dis 2009, 200:1509-1517.

32. Emerson PM, Ngondi J, Biru E, Graves PM, Ejigsemahu Y, Gebre T, Endeshaw T, Genet A, Mosher AW, Zerihun M, Messele A, Richards FO: Integrating an NTD with one of "the big three": combined malaria and trachoma survey in Amhara Region of Ethiopia. PLoSNegl Trop Dis 2008, 2:197.

33. Alemu A, Tsegaye W, Golassa L, Abebel G: Urban malaria and associated risk factors in Jimma town, south-west Ethiopia. Malar J 2011, 10:173.

34. Shargie EB, Gebre T, Ngondi J, Graves PM, Mosher AW, Emerson PM, Ejigsemahu Y, Endeshaw T, Olana D, WeldeMeskel A, Teferra A, Tadesse Z, Tilahun, Yohannes G, Richards FOJ: Malaria prevalence and mosquito net coverage in Oromia and SNNPR regions of Ethiopia. BMC Publ Health 2008, 8:321.

35. Mula P, Fernández-Martínez A, de Lucio A, Ramos JM, Reyes F, González V, Benito A, Berzosa P: Detection of high levels of mutations involved in anti-malarial drug resistance in Plasmodium falciparum and Plasmodium vivax at a rural hospital in southern Ethiopia. Malar $J$ 2011, 10:214.

36. Ashton RA, Kefyalew T, Tesfeye G, Pullan RL, Yadete D, Reithinger R, Kolaczinski JH, Brooker S: School-based surveys of malaria in Oromia Regional State, Ethiopia: a rapid survey method for malaria in low transmission settings. Malar J 2011, 10:25.

37. Abeku TA, van Oortmarssen GJ, Borsboom G, De Vlas SJ, Habbema JD: Spatial and temporal variations of malaria epidemic risk in Ethiopia: factors involved and implications. Acta Trop 2003, 87:331-340.

38. Yeshiwondim A, Gopal S, Hailemariam AT, Dengela DO, Patel HP: Spatial analysis of malaria incidence at the village level in areas with unstable transmission in Ethiopia. Int J Health Geogr 2009, 8:5.

39. Kiszewski $A E$, Tekelehaimanot $A$ : A review of the clinical and epidemiologic burdens of epidemic malaria. Am J Trop Med Hyg 2004, 71(Suppl 2):128-135.

40. Smith DL, Guerra CA, Snow RW, Hay SI: Standardizing estimates of the Plasmodium falciparum parasite rate. Malar J 2007, 6:131.

41. Giha HA, Rosthoj S, Dodoo D, Hviid L, Satti GM, Scheike T, Arnot DE: The epidemiology of febrile malaria episodes in an area of unstable and seasonal transmission. Trans R Soc Trop Med Hyg 2003, 94:645-651.

42. Oesterholt MJ, Bousema JT, Mwerinde OK, Harris C, Lushino P, Masokoto A, Mwerinde $\mathrm{H}$, Mosha FW, Drakeley CJ: Spatial and temporal variation in malaria transmission in a low endemicity area in northern Tanzania. Malar J 2006, 5:98.

43. WHO: Report on the malaria conference in equatorial Africa. Held under the joint auspices of the World Health Organization and of the commission for technical co-operation in Africa south of the Sahara.27 November-9 December, 1950. Kampala, Uganda. Geneva: World Health Organ Tech Rep Ser; 1951. $38: 72$.

doi:10.1186/1475-2875-11-199

Cite this article as: Santana-Morales et al.: Microscopy and molecular biology for the diagnosis and evaluation of malaria in a hospital in a rural area of Ethiopia. Malaria Journal 2012 11:199.

\section{Submit your next manuscript to BioMed Central and take full advantage of:}

- Convenient online submission

- Thorough peer review

- No space constraints or color figure charges

- Immediate publication on acceptance

- Inclusion in PubMed, CAS, Scopus and Google Scholar

- Research which is freely available for redistribution 